\title{
Variations in the Cochlear Implant Experience in Children With Enlarged Vestibular Aqueduct
}

\author{
Cedric Pritchett, MD, MPH; Teresa Zwolan, PhD; Farhan Huq, MD, MS; Amanda Phillips, BS; \\ Hemant Parmar, MBBS; Mohannad Ibrahim, MD; Marc Thorne, MD, MPH; Steven Telian, MD, MS
}

\begin{abstract}
Objectives/Hypothesis: To describe the clinical experience and characterize the outcomes of cochlear implantation (CI) in children with isolated enlarged vestibular aqueduct (IEVA) as compared to children with enlarged vestibular aqueduct (EVA) associated with other bony labyrinth abnormalities.

Study Design: Single, tertiary care, institutional retrospective review over 2 decades.

Methods: The clinical course and outcomes of 55 children with EVA undergoing CI between 1991 and 2013 were reviewed. Test measures included open and closed set speech perception tests, and various speech and language measures.

Results: In 18 children (32.7\%), IEVA was the only defect present. In 33 children (60\%), EVA occurred concomitantly with incomplete partition type 2 (IP 2) bilaterally, and three children with incomplete partition type 1 bilaterally. Ninety-two percent (51 of 55) occurred bilaterally and had matching bony defects. Mean age of CI was 73.4 months. A statistically significant defect-related and linguistic-status pattern was noted, impacting the timing of implantation: IEVA $=112.8$ months, IP $2=58.4$ months $(P<.001)$, prelingual deafness $=53.8$ months, postlingual deafness $=110.8$ months $(P<.001)$. Controlling for implant age and hearing loss severity, IEVA children demonstrated superior performance on speech perception tests (8.2 to 20.3 point differences), though statistical significance was inconsistent $(P=.01-.40$. Performance was also superior in speech and language tests, though statistical significance was never reached (2.9-13.9 point differences; $P=.14-.69$ ).

Conclusions: Children with hearing loss secondary to EVA respond meaningfully to cochlear implantation. However, the severity of temporal bone anomalies in these children has clinical relevance.
\end{abstract}

Key Words: Cochlear implant, language development, pediatric, congenital anomalies.

Level of Evidence: 4

Laryngoscope, 125:2169-2174, 2015

\section{INTRODUCTION}

An enlarged vestibular aqueduct (EVA) continues to be the most common radiographic abnormality apparent on temporal bone imaging studies of patients with congenital sensorineural hearing loss. ${ }^{1}$ Nearly 20 years

Additional Supporting Information may be found in the online version of this article.

From the Department of Otolaryngology-Head and Neck Surgery (c.P.), Northwestern University, Chicago, Illinois; Division of OtologyNeurotology, Department of Otolaryngology-Head and Neck Surgery (т.Z., S.т.), University of Michigan Health System, Ann Arbor, Michigan; Department of Otolaryngology-Head and Neck Surgery (F.H.), Henry Ford Health System, Detroit, Michigan; University of Michigan Medical School (A.P.), Ann Arbor, Michigan; Department of Radiology (M.I.), University of Michigan Health System, Ann Arbor, Michigan; Division of Pediatric Otolaryngology, Department of Otolaryngology-Head and Neck Surgery (м.т.), University of Michigan Health System, Ann Arbor, Michigan, U.S.A.

Editor's Note: This Manuscript was accepted for publication December 29, 2014.

Podium presentation at the Triological Annual Meeting at COSM, Las Vegas, Nevada, U.S.A., May 15-16, 2014.

Steven Telian, MD is on the medical advisory board of Cochlear Americas. Teresa Zwolan, $\mathrm{PhD}$, is on the audiology advisory board of Cochlear Americas and Med El.

The authors have no other funding, financial relationships, or conflicts of interest to disclose.

Send correspondence to Cedric V. Pritchett, MD, MPH Department of Otolaryngology-Head and Neck Surgery, Northwestern University, 155 E Superior St., Box 25, Chicago, IL 60611.

E-mail: cvprit.24@gmail.com

DOI: 10.1002/lary.25187 have passed since the first report of cochlear implantation (CI) in patients with an EVA. ${ }^{2}$ Since that time, the EVA syndrome itself has been better characterized. Originally emphasized for its progressive sensorineural hearing loss, it is now understood that it can encompass a heterogeneous course. ${ }^{1,3-7}$ Conductive components resulting in mixed losses may be identified, and the sensory component may be stable or fluctuating. ${ }^{1,5,8,9}$ Vestibular complaints are not uncommon. ${ }^{7,8,10}$ At least one known genetic cause has been discovered causing EVA, although the condition can be associated with syndromic or nonsyndromic hearing loss. ${ }^{11,12}$ Standardized protocols to reproducibly determine vestibular aqueduct size on temporal bone computed tomography (CT) have been developed. ${ }^{6,13}$ It is clear that EVA can exist in isolation or be accompanied by various temporal bone abnormalities (i.e., incomplete partition. It is largely accepted that EVA itself is not the causative factor of the hearing loss, but rather functions as a marker of an underlying abnormality, itself likely responsible for the hearing loss. ${ }^{7}$

In a similar manner, our understanding of the surgical management of EVA syndrome and the subsequent clinical outcomes has grown considerably. For example, surgical interventions to arrest hearing deterioration such as endolymphatic sac surgery have proven unsuccessful, $^{4,14}$ but CI for children meeting criteria has become the treatment of choice. Over time, surgical 
concerns such as perilymphatic "gushers," persisting cerebrospinal fluid leak, incomplete electrode insertion, and postoperative meningitis have been reported in a minority of patients. ${ }^{15}$ The child with EVA in isolation is expected to achieve postimplantation speech perception outcomes comparable to those of children with normal temporal bone anatomy. ${ }^{16,17}$ Even individuals with coexistent cochleovestibular abnormalities accompanying the EVA will generally have favorable postimplantation speech perception outcomes, although this is often dependent on the severity of the temporal bone deformity. ${ }^{15,16,18}$

Early implantation in children with congenital deafness is highly advantageous. ${ }^{19,20}$ Children are being implanted within the first year of their life. ${ }^{21}$ Despite the growing body of knowledge, ascertaining the best time for surgical intervention in the postlingually deaf child has been elusive. ${ }^{22}$ Substantial effort is invested in routinely following these children to intervene at the ideal time-when the hearing provided by a cochlear implant will reliably exceed that provided by amplification. Yet little information exists on speech and language outcomes of pediatric recipients of CI with EVA. A recent systematic review on cochlear implantation in children with cochleovestibular anomalies made no mention of postimplantation language skills. ${ }^{15}$

The University of Michigan has been performing CIs since 1984, and to date has performed more than 2,200 implant surgeries. Approximately $40 \%$ of the recipients have been children. This study details the experience over the past 2 decades of treating pediatric EVA CI recipients. Residual hearing, speech recognition, and speech/language skills of children with EVA who received a cochlear implant were systematically reviewed. Comparative analysis between children with isolated EVA (IEVA) and children with EVA accompanied by other bony labyrinth abnormalities was performed.

\section{MATERIALS AND METHODS}

University of Michigan Institutional Review Board approval was obtained for this project. Using medical records and a cochlear implant database, individuals who were 18 years of age or younger at time of EVA diagnosis and subsequently underwent CI during the years 1991 to 2013 were identified. The two neuroradiologist authors reviewed available CT temporal bone scans, and EVA diagnosis was confirmed by consensus. When images were unavailable for review, diagnosis was based upon careful review of radiology reports and otologist statements in the patient's medical record. In this study, the Cincinnati criteria for EVA was used; vestibular aqueducts measuring $>2 \mathrm{~mm}$ at the operculum or $>1 \mathrm{~mm}$ at the midpoint on axial CT were categorized as enlarged. ${ }^{6}$ Established criteria for classification of congenital inner ear malformation were applied. ${ }^{23}$ Incomplete partition type 2 (IP 2) consists of vestibular dilation and only 1.5 turns of the cochlea accompanying the EVA. Incomplete partition type 1 (IP 1 ) is characterized by a cystic cochlea with an absent modiolus and a dilated vestibule. Cochlear implantation candidacy was determined by measures that included pure-tone audiometry, speech perception and speech and language assessment, clinical exam, and radiographic imaging (i.e., CT).

Demographic data obtained included gender, age of implantation, age of activation, side of implantation, presence of a contralateral implant, implant type and quality of insertion. For sequential implants, data for the first implanted ear were recorded. A postimplantation communication method was noted and classified as auditory alone versus total communication; the former included children who utilized auditory-verbal or auditory-oral communication, whereas the latter included children who used any form of sign language (either alone or in combination with an oral mode of communication). Air and bone conduction thresholds were obtained using pure-tone audiometry. The most recent audiogram obtained prior to CI was used in data analyses. Pure-tone averages (PTAs) were calculated for each ear separately and were represented as the average threshold of $500 \mathrm{~Hz}, 1,000 \mathrm{~Hz}, 2,000 \mathrm{~Hz}$, and $3,000 \mathrm{~Hz}$. A "no response" at maximal testing for a particular frequency was categorically assigned a value of $125 \mathrm{~dB}$

Pre- and postoperative speech perception testing was achieved using four open-set tests: the Glendonald AuditoryScreening Procedure Sentences (GASP Sentences), the Glendonald Auditory-Screening Procedure Words (GASP Words), ${ }^{24}$ the Lexical Neighborhood Test and the Multisyllabic Lexical Neighborhood Test, ${ }^{25}$ and two closed set tests: the Word Intelligibility by Picture Identification test ${ }^{26}$ and the Northwestern University-Children's Perception of Speech ${ }^{27}$ test. Pre- and postoperative speech and language skills were assessed using three subtests of the Woodcock-Johnson (WJ) diagnostic battery ${ }^{28}$ : Oral Comprehension, Passage Comprehension (WJPC), and Understanding Directions. Additionally, the Peabody Picture Vocabulary test, ${ }^{29}$ which examines receptive vocabulary, and the Expressive Vocabulary Test, ${ }^{30}$ which evaluates expressive vocabulary, were also administered. Standardized scoring is used for all of the speech and language measures; a score falling between 85 and 115 is considered within normal range. The resulting data were organized into the following clustered postoperative increments: 6 months (3-9 months), 12 months (9-18 months), and 24 months (18-30 months). All available data were used. Patients with cognitive impairment or inconsistent users of their CI were excluded.

Statistical analysis was performed using available software. A two-sided Student $t$ test was used on continuous level data; $\chi^{2}$ analysis was performed on categorical level data. Linear models fit using generalized estimating equations (GEEs) were used to assess the relationships between predictors (defect type, age at implant, severity of hearing loss, and postoperative interval) and outcomes (various speech and language tests). Age at implantation was dichotomized to $<5$ years and $\geq 5$ years of age. Hearing loss severity was classified as severe (70-90 dB) or profound $(>90 \mathrm{~dB})$ in the better hearing ear. GEE accounts for the dependency between repeated measures taken on individual subjects and was applied using compound symmetric withinsubject covariance. Mean structures were defined in terms of main effects for the three predictors of interest. No formal adjustments for multiple comparisons were performed, so significance levels were interpreted conservatively.

\section{RESULTS}

\section{Demographics}

Fifty-seven patients with a diagnosis of EVA underwent cochlear implantation from 1991 to 2013. Two patients were excluded, as coexistent reasons for congenital deafness could not be excluded (Connexin 26 with homozygous 35delG defects for one patient and congenital cytomegalovirus in the other). Demographic information for the remaining 55 individuals is provided Table I). Fifty-one of 55 subjects demonstrated 


\begin{tabular}{|c|c|c|c|}
\hline \multicolumn{4}{|c|}{$\begin{array}{l}\text { TABLE I. } \\
\text { Demographics of Patients With EVA Undergoing Cochlear } \\
\text { Implantation. }\end{array}$} \\
\hline & & SD & $P$ Value \\
\hline Total patients & 55 & & \\
\hline Male & 27 & & \\
\hline Female & 28 & & \\
\hline Bilateral & 52 & & \\
\hline Unilateral & $3[\text { right }=2 \text {; left }=1]^{*}$ & & \\
\hline EVA alone (total) & 18 & & \\
\hline Available & 15 & & \\
\hline IP 2 & $34 \dagger$ & & \\
\hline Available & 33 & & \\
\hline IP 1 & $3^{\dagger}$ & & \\
\hline Available & 2 & & \\
\hline \multicolumn{4}{|c|}{ Immediate preoperative PTA (range) } \\
\hline Right & $103.3(71-121.5)$ & 13.55 & .26 \\
\hline Left & $100.2(68-125)^{\ddagger}$ & 14.09 & \\
\hline EVA alone & $98.7(77-111)$ & 11.42 & .03 \\
\hline IP 2 & $105.2(71-125)$ & 13.71 & \\
\hline IP 1 & 109.1 & & \\
\hline \multicolumn{4}{|c|}{ Defect type preoperative PTA (range) } \\
\hline EVA right, $n=13$ & $98.8(77-113.1)$ & 10.28 & .94 \\
\hline EVA left, $n=13$ & $98.6(68-115)$ & 12.82 & \\
\hline IP 2 right, $n=27$ & $107.6(71-125)^{\star}$ & 13.67 & .10 \\
\hline IP 2 left, $n=27$ & $102.8(72-125)^{\star}$ & 13.56 & \\
\hline IP 1 right, $n=2$ & $112.5(110-115)$ & & \\
\hline IP 1 left, $n=2$ & $110.1(108-112)$ & & \\
\hline \multicolumn{4}{|c|}{ Age implantation, mean age, mo } \\
\hline Overall mean & 73.4 & 53.23 & \\
\hline$E V A, n=13$ & 112.8 & 57.42 & $<.001$ \\
\hline IP $2, n=29$ & 58.4 & 44.1 & \\
\hline IP $1, n=2$ & 74 & & \\
\hline Severe $(\mathrm{dB}<90)$ & 67.9 & 37.06 & .47 \\
\hline Profound $(\mathrm{dB}>90)$ & 80.2 & 58.8 & \\
\hline Auditory only & 75 & 52.97 & .92 \\
\hline Combined communication & 76.9 & 56.99 & \\
\hline \multicolumn{4}{|c|}{ Postimplant communication method, total no } \\
\hline Auditory only & 30 & & \\
\hline Combined & 12 & & \\
\hline
\end{tabular}
bones.

${ }^{*}$ Three patients with EVA only and normal contralateral temporal

${ }^{\dagger}$ Note: one patient with IP 1 and IP 2.

¥Used to denote limit of audiometry with no response.

$E V A=$ enlarged vestibular aqueduct; IP = incomplete partition; $\mathrm{PTA}=$ pure-tone average; SD = standard deviation.

symmetrically matched temporal bone anatomy. Three patients had unilateral EVA with normal contralateral temporal bone anatomy, and one patient had an IP 2 of the right ear and IP 1 of the left ear. There were no statistically significant differences in the distribution of children when comparing various combinations of radiologic defect, degree of residual hearing, or communication categories as detailed in Table II. Implantation characteristics of the recipients including laterality and device type are provided in Table III.
TABLE II.

Classifying Characteristics of Children With Enlarged Vestibular Aqueduct Undergoing Cochlear Implantation.

\begin{tabular}{lcc}
\hline & No. & $P$ Value \\
\hline Defect type vs residual hearing & & .72 \\
EVA + severe & 4 & \\
EVA + profound & 10 & \\
IP 2 + severe & 7 & \\
IP 2 + profound & 23 & .46 \\
Communication method vs. residual hearing & & \\
Auditory + severe & 9 & \\
Auditory + profound & 19 & \\
Total + severe & 2 & \\
Total + profound & 9 &
\end{tabular}

EVA = enlarged vestibular aqueduct; IP = incomplete partition;

\section{Audiometric Outcomes}

Preoperative audiograms were available for 51 children. No significant difference existed in the preoperative mean PTAs for the right and left ears of the group collectively $(P=.26)$. However, ears with more complex temporal bone defects demonstrated greater hearing loss (higher PTAs) at the time of surgery $(P=.03)$. The mean age at implantation for children with IEVA was 112.8 months; the mean of children with IP 2 anatomy was 58.4 months $(P<.001)$. The small number of patients with IP 1 and the large range in age at implantation make meaningful interpretation difficult for this group. The PTA at time of implantation, grouped by decade, was also explored. A trend toward implantation with more residual hearing was noted with each progressive

\begin{tabular}{|c|c|}
\hline \multicolumn{2}{|c|}{$\begin{array}{c}\text { TABLE III. } \\
\text { Implantation Characteristics of Enlarged Vestibular Aqueduct } \\
\text { Recipients. }\end{array}$} \\
\hline Total implants & 55 \\
\hline \multicolumn{2}{|l|}{ Implant type } \\
\hline (Cl22M) Nucleus 22 & 7 \\
\hline (Cl24M) Nucleus 24M & 9 \\
\hline (Cl24R) Nucleus 24 contour & 13 \\
\hline (Cl24RE) Nucleus Freedom contour advance & 13 \\
\hline (Cl 500 series) Nucleus CI 512 & 4 \\
\hline Advanced Bionics HiRes 90K & 2 \\
\hline Advanced Bionics Clarion & 5 \\
\hline Med-El Combi 40 \pm & 2 \\
\hline \multicolumn{2}{|l|}{ Implant laterality } \\
\hline Right & 37 \\
\hline Left & 18 \\
\hline Subsequent contralateral (or bilateral) & 9 \\
\hline \multicolumn{2}{|l|}{ Insertion } \\
\hline Device failure, reimplant & 6 \\
\hline Full & 52 \\
\hline Partial & 1 \\
\hline Unknown & 2 \\
\hline
\end{tabular}

Pritchett et al.: Cochlear Implantation in EVA Children 
TABLE IV.

Pediatric EVA Cochlear Implantation Regression Model $^{*}$

\begin{tabular}{|c|c|c|c|c|c|c|c|c|c|}
\hline & \multicolumn{3}{|c|}{ Defect Type, IP 2 vs. EVA } & \multicolumn{3}{|c|}{ Age at Implant, $>5$ Years vs. $<5$ Years } & \multicolumn{3}{|c|}{ Severity of Loss, Profound vs. Severe } \\
\hline & $\begin{array}{c}\text { Point } \\
\text { Difference }\end{array}$ & $P$ Value & $95 \% \mathrm{Cl}$ & $\begin{array}{c}\text { Point } \\
\text { Difference }\end{array}$ & $P$ Value & $95 \% \mathrm{Cl}$ & $\begin{array}{c}\text { Point } \\
\text { Difference }\end{array}$ & $P$ Value & $95 \% \mathrm{Cl}$ \\
\hline \multicolumn{10}{|c|}{ Speech perception } \\
\hline GASP sentence & $-20,3$ & $.03^{\dagger}$ & -39.2 to -1.4 & 26.9 & $<.01^{\dagger}$ & 9.1 to 44.6 & 12 & .2 & 29.2 to 5.3 \\
\hline GASP word & -11.9 & .22 & -31.3 to 7.6 & 2.3 & $.02^{\dagger}$ & 2.5 to 38.1 & -13.5 & $.05 \dagger$ & -27.8 to -0.01 \\
\hline LNT & -11.8 & .16 & -28.5 to 4.8 & -9.5 & .42 & -33.1 to 14.1 & -2.7 & .73 & -18.5 to 13.0 \\
\hline MLNT & -9.2 & .4 & -31.1 to 12.7 & -0.1 & 1 & -23.6 to 23.4 & -10 & .22 & -26.3 to 6.24 \\
\hline NU-CHIPS & -8.2 & .27 & -22.9 to 6.5 & 3 & .67 & -10.9 to 16.9 & -0.9 & .89 & -13.9 to 12.1 \\
\hline WIPI & -16.6 & $.01^{\dagger}$ & -26.5 to -6.8 & -4.4 & .52 & -18.0 to 9.2 & -6.3 & .34 & -19.7 to 7.0 \\
\hline \multicolumn{10}{|c|}{ Speech and language } \\
\hline PPVT & -3 & .68 & & 8.4 & .21 & -5.0 to 21.7 & 6.9 & $.03 \dagger$ & 38.8 to 99.7 \\
\hline WJOC & -10 & .46 & & -15.7 & .22 & -41.2 to 9.7 & 20.2 & .23 & -13.3 to 53.7 \\
\hline WJPC & 3.3 & .22 & & -10.6 & .58 & -49.3 to 28.1 & -2.8 & .56 & -12.5 to 6.8 \\
\hline WJUD & -8.9 & .48 & & -4.3 & .7 & -27.0 to -18.3 & 27.4 & .11 & -7.1 to 61.9 \\
\hline EVT & -13.4 & .16 & & 0.15 & .99 & -16.6 to 16.9 & 8.4 & $<.01 \dagger$ & 2.2 to 14.7 \\
\hline
\end{tabular}

*Differences in mean speech perception and mean speech/language scores are provided for comparisons made between the following groups: 1) defect type (EVA vs. IP 2), 2) age at implant (children implanted $>5$ years of age versus $<5$ years of age, and 3 ) severity of loss (children with severe losses vs children with profound losses). Negative point value differences reflect a higher mean score by the second group. Values reflect points in score and are adjusted for postimplantation status. Significant differences between the two groups are compared.

${ }^{\dagger} P \leq .05$.

$\mathrm{Cl}=$ confidence interval; $\mathrm{EVA}=$ enlarged vestibular aqueduct; $\mathrm{EVT}=$ Expressive Vocabulary Test: GASP = Glendonald Auditory Screening Procedure; IP = incomplete partition; LNT = Lexical Neighborhood Test; MLNT = Multisyllabic Lexical Neighborhood Test; NU-CHIPS = Northwestern University-Children's Perception of Speech; PPVT = Peabody Picture Vocabulary Test; WIPI = Word Intelligibility by Picture Identification; WJOC = Woodcock Johnson Oral Comprehension; WJPC = Woodcock Johnson Passage Comprehension WJUD = Woodcock Johnson Understanding Directions.

decade (see Supporting Figure 1 in the online version of this article).

\section{Speech Perception}

Speech perception data were available for 42 children; 16 with IEVA (10 profound and six severe hearing loss), and 26 with IP 2 (20 profound and six severe) (see Supporting Figure 2A,B in the online version of this article, which demonstrate the clinical course for all subjects [mean scores are shown]). Statistically significant improvement over the baseline $(P<.001-.05)$ was achieved. Children in both categories demonstrated the steepest slope in hearing recovery during the first 6 months following implantation, but continued to improve or remained stable during the study period. The exception to this trend in the separated groups appeared in the IEVA category, where a decline in GASP word and sentence testing was noted at the 2-year mark. Dataset analysis in this case suggested this was a function of the small number of individuals in this category at this interval, and marginal performance in one of three individuals affected the mean considerably.

\section{Language}

Speech and language data for 41 children were available for analysis; 14 with IEVA (eight profound and six severe), and 27 with IP 2 (20 profound, five severe, two unclassified). Mean postimplant scores were similar, remaining stable or slightly improving over baseline (for results see Supporting Figure 3A,B in the online version of this article).

\section{Model}

Depending on the assessment tool, scores for 15 to 30 children (speech perception) and 12 to 34 children (speech and language) were included in the regression model. IEVA children consistently outperformed their IP 2 counterparts in speech perception tests. Controlling for age and severity of hearing loss at implantation, there was an 8 to 20-point difference between the groups, though statistically significant in less than half (Table IV). A similar performance discrepancy was seen in speech and language assessment, although this difference was not statistically significant. (The exception in WJPC was attributed to the insufficient sample size.) A non-statistically significant advantage was noted in children with severe losses, compared to profound loss of varying magnitudes in both types of tests. $\mathrm{P}=.05$ in the GASP Word testing. Older children demonstrated a meaningful performance advantage in two of the openset tests (GASP Sentence and Word: 26.9, $P<.01$; and 20.3, $P=.02$, respectively), after controlling for defect type and severity of hearing loss.

\section{DISCUSSION}

The enlarged vestibular aqueduct syndrome is a frequent cause for sensorineural hearing loss in early childhood. However, children with an EVA are a heterogeneous group. Existing literature demonstrates 
that if an EVA occurs in isolation, the post-cochlear implant hearing outcomes should be similar to those of a child with normal temporal bone anatomy. ${ }^{16}$ However, EVA occurs concomitantly with other cochleovestibular anomalies in at least $40 \%$ of cases. ${ }^{10,31}$ The purpose of this study was to characterize both speech perception and speech/language measures within this group following CI.

An anatomical approach to EVA classification remains among the oldest and most popular. ${ }^{10}$ The established classification of cochleovestibular malformation detailed by Sennaroglu and Saatci $^{23}$ was used to group our children into IEVA, IP 2 (classic Mondini deformity), and IP 1 categories. Isolated EVA and IP 2 are considered mild to moderate, whereas IP 1 is considered a severe deformity. Our study explored all children with the EVA who received CI, but did not include defects occurring in the absence of an enlarged vestibular aqueduct. The findings appear consistent with published results from others, as only a minority had severe temporal bone defects. ${ }^{15}$

As a measure of residual hearing, the PTA in the better hearing ear was identified. Over a quarter of the patients in our cohort received CI with only severe hearing loss. These children were distributed across the duration of the study, though we noted a measurable increase in the number of children with severe losses and a decrease in the number of children with profound losses who received CI in the latter decades. Ninety-five percent of children implanted during the first decade had profound hearing loss, $62.5 \%$ in the second decade, and $60 \%$ thus far in the third decade. These findings are likely due to growing awareness that children with EVA are placed at a disadvantage when CI surgery is delayed until profound hearing loss occurs, combined with an increased awareness over time of the improved hearing provided by CI. Accordingly, our model supports this, and demonstrated at 1- to 13.5-point improved performance in children implanted with severe compared to profound hearing loss. Statistical significance was present only in one speech perception and two speech and language tests, but this likely reflects our small sample size. This finding can be further evaluated once additional children with EVA receive an implant soonerwhen an implant is provided prior to the hearing loss reaching a profound level.

The mean age at implant in this study was just over 6 years of age (73.4 months), well within what is reported in the literature. ${ }^{2,18,22,31-34}$ This varied considerably based on defect type. Children with IEVA were substantially older at the time of implantation than their peers, who presented with EVA combined with other inner ear structural defects (112.8 months vs. 58.4 months, $P<.001)$. Such findings are not surprising, as one would expect more complex abnormalities to positively correlate with progressive hearing loss. In reviewing the literature, no large study reporting that the EVA anomaly affected age of implantation could be identified. Lee et al. suggested more severe hearing impairment secondary to development defects as a partial explanation for the inferior performance of children with additional anomalies accompanying the EVA on postimplant PTA testing. ${ }^{32}$ In their published series of 23 patients with EVA, they reported concomitant temporal bone anomalies in $12(52 \%)$ of their patients. Their mean age of implantation was 5.3 years, but they did not compare the age at implant for the two groups.

The greatest period of speech perception improvement in our cohort occurred within the first 12 months following implantation. Though both groups experienced meaningful postimplantation gains, children with IEVA tended to demonstrate stronger speech perception skills prior to and following CI than their IP 2 counterparts. We attribute this to the superior language skills this group had prior to implantation. In our study, implantation enabled nearly all children to improve their speech and language skills over time, with many obtaining scores comparable to children with normal hearing. Speech and language evaluations should therefore be carefully followed overtime, and strongly considered in determining when intervention with a cochlear implant should occur.

\section{Limitations}

The retrospective nature of this study limited the completeness of the data available. We could not report on onset or duration of deafness in this group. It is quite feasible that a child with rapidly progressive hearing loss adapts differently than a child who experiences a fluctuating or slowly deteriorating decline in hearing. We also could not investigate the age at which initial amplification was provided or the effects of sequential or bilateral implantation in this population. To date, a total of nine children have received bilateral implants, warranting further investigation of this phenomenon when this group of children is larger. The inability of younger subjects to perform certain tests requires the administration of different test batteries based upon the age and language skills of the child (often varying in the 24month span covered by this study). This factor hindered our ability to conclusively evaluate postimplantation speech and language outcomes related to the degree of residual hearing. The paucity of such data in the published literature on this topic makes it apparent that longitudinal data are difficult to acquire despite robust efforts to ensure regular patient follow-up. Given the substantial time span of this study, we did encounter constraints due to changes in technology. Archived radiographic images obtained prior to 2000 were sometimes unavailable.

Over time, several variables have emerged as predictors of language achievement after CI, including age at implantation, shorter periods of hearing loss, greater residual hearing at the time of surgery, higher ratings of parent-child interactions, higher socioeconomic status, communication method, presence of additional disabilities, and multilingualism. ${ }^{35}$ Language development is complex, and the measureable benefit of interventions is known to be quite variable. Ganek et $\mathrm{al}^{36}$ cautioned that the wide variability in language outcomes and large standard deviations limit the ability to construct an 
average implant recipient. This is especially true when investigating outcomes in children with dysmorphic temporal bones. Despite these limitations, we believe the classification of pediatric CI recipients as performed in this study adds meaningfully to the existing literature.

\section{CONCLUSION}

It has previously been established that the rate of speech perception progression and the maximal open-set response achieved with the use of cochlear implants are related to the severity of the inner ear malformation. ${ }^{16,18}$ The current study indicates this is also the case when treating children with enlarged vestibular aqueducts. On average, children with isolated EVA have aided hearing benefit for a longer period of time prior to implantation than their IP 2 counterparts. Both groups respond very favorably to $\mathrm{CI}$, though there does appear to be a performance advantage for children with isolated EVA. The challenge of producing long-term data for speech perception outcomes persists. Accrual of data with the intent to increase our understanding of the relationship between recovered hearing and ongoing linguistic development should continue.

\section{Acknowledgments}

The authors give special thanks to Kerby Shedden, $\mathrm{PhD}$, and the University of Michigan Center for Statistical Consultation and Research for their assistance in this project.

\section{BIBLIOGRAPHY}

1. Madden C, Halsted M, Benton C, Greinwald JH, Choo DI. Enlarged vestibular aqueduct syndrome in the pediatric population. Otol Neurotol 2003;24:625-632.

2. Slattery WH, Luxford WM. Cochlear implantation in the congenital malformed cochlea. Laryngoscope 1995:105:1184-1187.

3. Valvassori GE. The large vestibular aqueduct and associated anomalies of the inner ear. Otolaryngol Clin North Am 1983;16:1, 95-101.

4. Jackler RK, De La Cruz A. The large vestibular aqueduct syndrome. Laryngoscope 1989;99:1238-1242.

5. Colvin IB, Beale T, Harrops-Griffths K. Long-term follow-up of hearing loss in children and young adults with enlarged vestibular aqueducts: relationship to radiologic findings and Pendred Syndrome diagnosis. Laryngoscope 2006;116:2027-2036.

6. Boston M, Halstead M, Meinzen-Derr J, et al. The large vestibular aqueduct: a new definition based on audiologic and computed tomography correlation. Otolaryngol Head Neck Surg 2007;136:972-977.

7. Gopen Q, Zhou G, Whittenmore K, Kenna M. Enlarged vestibular aqueduct: review of controversial aspects. Laryngoscope 2011;121:1971-1978.

8. Grimmer JF, Hedlund G. Vestibular symptoms in children with enlarged vestibular aqueduct anomaly. Int $J$ Pediatr Otorhinolaryngol 2007;71: $275-282$

9. Lai CC, Shaio AS. Chronological changes of hearing in pediatric patients with large vestibular aqueduct syndrome. Laryngoscope 2004;114:832-838.
10. Valvassori GE, Clemis JD. The large vestibular aqueduct syndrome. Laryngoscope 1978;88:723-728.

11. Abe S, Usami S, Hoover D, et al. Fluctuating sensorineural hearing loss associated with enlarged vestibular aqueduct maps to $7 \mathrm{q} 31$, the region containing the Pendred gene. Am J Med Genet 1999;82:322-328.

12. Usami S, Abe S, Weston M, et al. Non-syndromic hearing loss associated with enlarged vestibular aqueduct is caused by PDS mutations. Hum Genet 1999;104:188-192.

13. Dewan K, Wippold FJ, Lieu JEC. Enlarged vestibular aqueduct in pediatric sensorineural hearing loss. Otolaryngol Head Neck Surg 2009;140: $552-558$.

14. Welling C, Slater P, Martyn M, et al. Sensorineural hearing loss after occlusion of the enlarged vestibular aqueduct. Am J Otol 1999;20:338343.

15. Pakdaman MN, Hermann BS, Curtin HD, Van Beek-King J, Lee DJ. Cochlear implantation in children with anomalous cochleovestibular anatomy: a systematic review. Otolaryngol Head Neck Surg 2012;146:180 190.

16. Papsin BC. Cochlear implantation in children anomalous cochleovestibular anatomy. Laryngoscope 2005;115(suppl 106):1-26.

17. Miyamoto RT, Bichey BG, Wynne MK, Kirk KI. Cochlear implantation with large vestibular aqueduct syndrome. Laryngoscope 2002;112:11781182.

18. Buchman CA, Copeland BJ, Yu KK, Brown CJ, Carrasco VN, Pillsbury HC. Cochlear implantation in children with congenital inner ear malformations. Laryngoscope 2004;114:309-316.

19. Geers A, Tobey E, Moog J, Brenner C. Long-term outcomes of cochlear implantation in the pre-school years: from elementary grades to high school. Int J Audiol 2008;47(suppl 2):S21-S30.

20. Fulcher A, Purcell AA, Baker E, Munro N. Listen up: children with early identified hearing loss achieve age-appropriate speech/language outcomes by 3 years of age. Int $J$ Pediatr Otorhinolaryngol 2012;76:17851794.

21. Cosetti M, Roland JT Jr. Cochlear implantation in the very young child: issues unique to the under-1 population. Trend Amplif 2010;14:46-57.

22. Vassoler TMF, Bergonse GFR, Junior MS, Bevilacqua MC, Filbo OAC Cochlear implant and large vestibular aqueduct syndrome in children. Rev Bras Otorrinolaringol 2008;74:260-264.

23. Sennaroglu L, Saatci I. A new classification for cochleovestibular malformations. Laryngoscope 2002;112:2230-2241.

24. Erber NP. Auditory Training. Washington, DC: Alexander Graham Bell Association for the Deaf; 1982.

25. Kirk KI, Pisoni DB, Osberger MJ. Lexical effects on spoken word recognition by pediatric cochlear implant users. Ear Hear 1995;16:470-481.

26. Ross M, Lerman J. Word Intelligibility by Picture Identification. Pitts burgh, PA: Stanwix House; 1979.

27. Elliott L, Katz D. Development of a New Children's Test of Speech Discrimination. St. Louis, MO: Auditec; 1980.

28. Woodcock RW, McGrew KS, Mather N. Woodcock Johnson III Tests of Achievement. Rolling Meadows, IL: Riverside; 2007.

29. Dunn L, Dunn D. Peabody Picture Vocabulary Test. Minneapolis, MN: Pearson; 2007.

30. William K. Expressive Vocabulary Test 2. Circle Pines, MN: American Guidance Service; 2007.

31. Au G, Gibson W. Cochlear implantation in children with large vestibular aqueduct syndrome. Am J Otol 1999;20:183-186.

32. Lee KH, Lee J, Isaacson B, Kutz W, Roland PS. Cochlear implantation in children with enlarged vestibular aqueduct. Laryngoscope 2010;120: $1675-1681$.

33. Fahy CP, Carney AS, Nikolopoulos TP, Ludman CN, Gibbin KP. Cochlear implantation in children with large vestibular aqueduct syndrome and a review of the syndrome. Int J Pediatr Otorhinolaryngol 2001;59:207215.

34. Harker LA, Vanderheiden S, Veazey D, Gentile N, McCleary E. Multichannel cochlear implantation in children with large vestibular aqueduct syndrome. Ann Otol Rhinol Laryngol 1999;108:39-43.

35. Niparko JK, Tobey EA, Thal DJ, et al. Spoken language development in children following cochlear implantation. JAMA 2010;303:1498-1506.

36. Ganek H, Robbins AM, Niparko JK. Language outcomes after cochlear implantation. Otolaryngol Clin N Am 2012;45:173-185. 